\title{
Modification of Even-A Nuclear Mass Formula
}

\author{
Jingyi Zhang \\ Department of Physics, University of Shanghai for Science and Technology, Shanghai, China \\ Email: jingchyi@sina.cn
}

How to cite this paper: Zhang, J.Y. (2017)

Modification of Even-A Nuclear Mass Formula. Journal of Applied Mathematics and Physics, 5, 2302-2310.

https://doi.org/10.4236/jamp.2017.511187

Received: October 24, 2017

Accepted: November 26, 2017

Published: November 29, 2017

Copyright $\odot 2017$ by author and Scientific Research Publishing Inc. This work is licensed under the Creative Commons Attribution International License (CC BY 4.0).

http://creativecommons.org/licenses/by/4.0/

\section{(c) (i) Open Access}

\begin{abstract}
In this paper we obtain an empirical mass formula of even-A nuclei based on residual proton-neutron interactions. The root-mean-squared deviation (RMSD) from experimental data is at an accuracy of about $150 \mathrm{Kev}$. While for heavy nuclei, we give another formula that fits the experimental data better (RMSD $\approx 119 \mathrm{Kev})$. We have successfully described the experimental data of nuclear masses and predicted some unknown masses (like ${ }^{200}$ Ir not involved in AME2003, the deviation of our predicted masses from the value in AME2012 is only about $82 \mathrm{keV}$ ). The predictive power of our formula is more competitive than other mass models.
\end{abstract}

\section{Keywords}

Residual Proton-Neutron Interactions, Nuclear Masses, Binding Energies

\section{Introduction}

The study of nuclear masses and energy levels has always been one of the most challenging frontiers in the field of nuclear physics. There are two types to describe and understand the nuclear masses, one of which is global relations, and the other is local. Some global nuclear mass models such as Weizäscker model [1], Duflo-Zuker model [2], the finite range droplet model [3], a recent macroscopic-microscopic mass formula [4] [5] [6] etc., successfully produce the measured masses with accuracy at the level of $300-600 \mathrm{Kev}$. However, the global mass models require more physics and more information about nuclear force to get better description of the nuclear masses. On the other hand, the local mass relations, such as the isobaric multiplet mass equation (IMME), the Garvey-Kelson (GK) relations, which use the predicted nuclear masses and the residual proton-neutron interactions to evaluate the mass. It is found that the local mass relations are just approximately satisfied in known masses, so it has a good potential to predict the unknown masses. 
In this paper, our purpose is to obtain a residual proton-neutron interactions formula of even-A nuclei from those of neighboring nuclei. In Section II we introduce the residual proton-neutron interactions and obtain our formula based on the proton-neutron interactions between the last proton and the last neutron. Then we introduce two modifications to improve our formula. The RMSD from experimental data is about $150 \mathrm{Kev}$. And for heavy nuclei, we obtain another formula fits with the experimental data even more precise. With our further refinement of heavy nuclei, the RMSD gets even smaller to about $120 \mathrm{Kev}$. In Section III we successfully predict some unknown masses. The result shows that the predict power of our formula is competitive with others. In Section IV we discuss and summarize the results of this paper.

\section{The Residual Proton-Neutron Interactions}

The residual proton-neutron interaction plays an important role in the evolution of collective, deformation and phase transition [7] [8] [9] [10], so it has attracted many attentions [11]-[17]. The proton-neutron interactions between the last $i$ protons and $j$ neutrons is given by

$$
V_{i p-j n}(Z, N)=B(Z, N)+B(Z-i, N-j)-B(Z, N-j)-B(Z-i, N) .
$$

The famous formula GKL and GKT were derived from the neutron-proton interactions between the last neutron and proton [18] [19]. The relationship between Garvey-Kelson quality is a semi empirical relationship between 6 adjacent nuclear mass. If the interaction between neighboring nuclei changes slowly in the local range, it can be completely counteracted by the addition and subtraction of many adjacent nuclei. Garvey-Kelson mass relationship has two common relationships:

$$
\begin{aligned}
& M(N, Z+1)+M(N-1, Z-1)+M(N+1, Z) \\
& -M(N, Z-1)-M(N-1, Z)-M(N+1, Z+1)=0, \\
& M(N, Z-1)+M(N-1, Z+1)+M(N+1, Z) \\
& -M(N, Z+1)-M(N-1, Z)-M(N+1, Z-1)=0,
\end{aligned}
$$

where $M(N, Z)$ denotes the mass of a nucleus with neutron number $N$ and proton number $Z$. Equation (2) is called the longitudinal Garvey-Kelson relation (GKL), and Equation (3) the transverse (GKT).

In this section, we use the residual proton neutron interactions between the last proton and the last neutron to form our formula. According to the Equation (1), it is easy to obtain that the residual proton-neutron interactions between the last proton and the last neutron is defined as

$$
\begin{aligned}
V_{1 p-1 n}(Z, N) & =B(Z, N)+B(Z-1, N-1)-B(Z, N-1)-B(Z-1, N) \\
& =M(Z, N)+M(Z-1, N-1)-M(Z, N-1)-M(Z-1, N)
\end{aligned}
$$

The Garvey-Kelson mass relations require six nuclei, but our formula requires only four. So our formula involves less number of nuclei, its predictions in iterative extrapolations is the more reliable, and its deviations are smaller in the 
extrapolation process.

In recent years, many papers tried to find formulas to describe and evaluate the nuclear masses, but many of them have a large RMSD. In this work, we focus on the even-A nuclei, through the study on the neighboring nuclei with the database in AME2012 [20].

For the residual nuclear proton-neutron interactions which $A \geq 42$, we calculate the $\delta V_{1 p-1 n}$ as shown in Figure 1. Based on that, we empirically obtained the residual proton-neutron interactions formula of even-A nuclei. The formula is as follows:

$$
\begin{aligned}
\overline{\delta V_{1 p-1 n}} & =B(Z, N+1)+B(Z-1, N)-B(Z, N)-B(Z-1, N+1) \\
& \cong \frac{515.6}{A^{2}}+\frac{62.78}{A}+0.1079 \mathrm{keV}
\end{aligned}
$$

$\overline{\delta V_{1 p-1 n}}$ is the average values of $\delta V_{1 p-1 n}$ for nuclei with the same mass number $A$.

We find that the average binding energy of our predicted mass agrees well with the specific binding energy curve. We successfully describe and predict some even-A nuclear masses by using these equations and some known experimental nuclear masses in AME2012 for calculation of $\delta V_{1 p-1 n}$.

It can be seen from the Figure 1 that the interaction of proton-neutron is

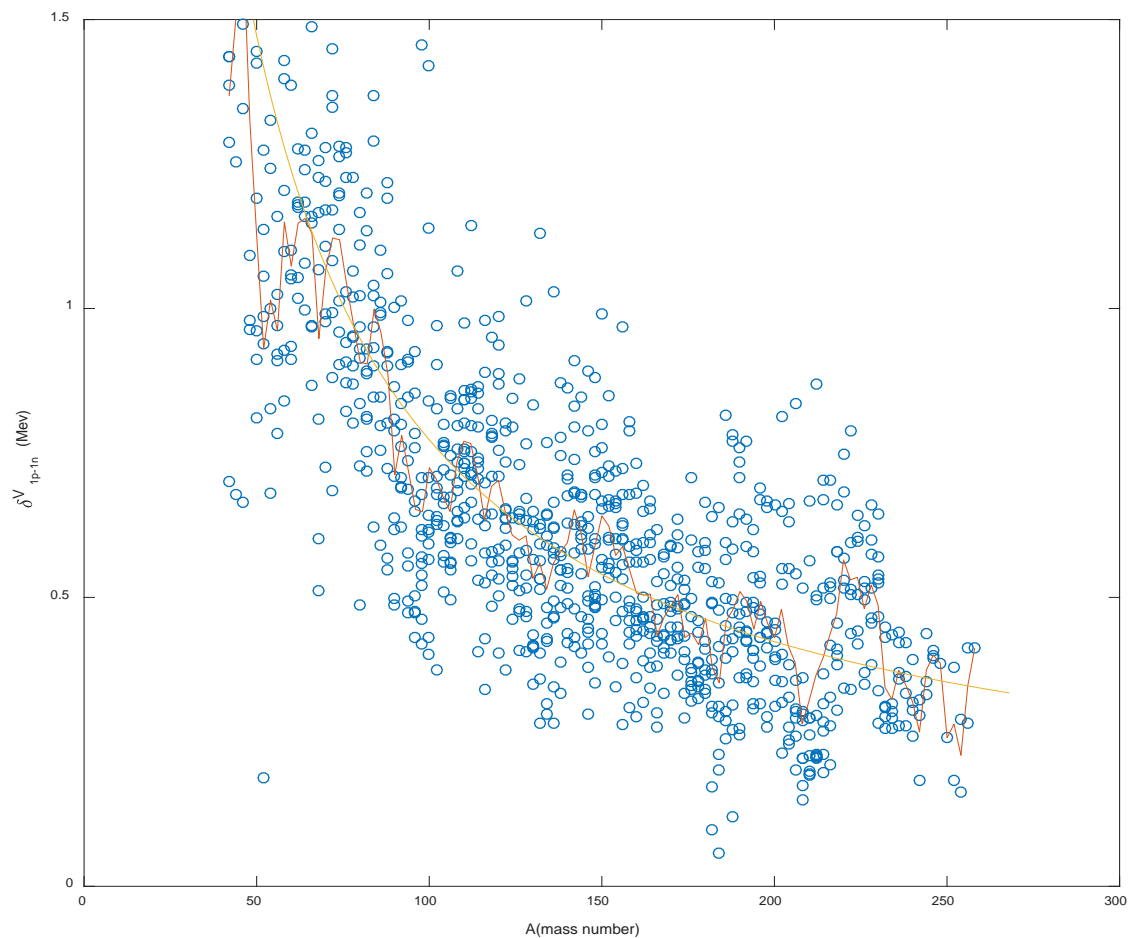

Figure 1. Circles show that the residual proton-neutron interactions $\delta V_{1 p-1 n}$. The curve is plotted by using the average values of $\delta V_{1 p-1 n}$ for nuclei with the same mass number $A$, expressed as $\overline{\delta V_{1 p-1 n}}$. The smoothed curve are plotted in terms of equation $\overline{\delta V_{1 p-1 n}}(A)=\frac{515.6}{A^{2}}+\frac{62.78}{A}+0.1079 \mathrm{keV}$ for even-A nuclei with $A \geq 42$. 
more stable in the heavy nuclei region than in the light nuclei region.

In order to better describe the quality of the nucleus, we will improve the above formula with some amendments, donated by $\delta V_{1 p-1 n}^{c a l}$ as the final improvement results [4] [5] [6]. The first is called the Coulomb correction, denoted by $\Delta_{C}$ :

$$
\Delta_{C}(Z, N) \approx a_{C}\left(-\frac{4}{9} Z^{4 / 3} A^{-7 / 3}-\frac{2}{3} Z A^{-4 / 3}+\frac{4}{9} Z^{2} A^{-7 / 3}+\frac{4}{9} Z^{1 / 3} A^{-4 / 3}\right),
$$

the second is called the symmetry energy correction, denoted by $\Delta_{\text {sym }}$ :

$$
\Delta_{\text {sym }}(Z, N)=a_{\text {sym }} \frac{1}{A(2+|I A|)^{3}}+b_{\text {sym }} A^{-1},
$$

where $I=(N-Z) / A$ and $a_{C}=10.51, a_{\text {sym }}=20126, b_{\text {sym }}=-61.25$ as parameters [17] [21].

The revised $\delta V_{1 p-1 n}(Z, N)$ is as follows:

$$
\delta V_{1 p-1 n}^{c a l}(Z, N)=\overline{\delta V_{1 p-1 n}}-\Delta_{C}(Z, N)-\Delta_{\text {sym }}(Z, N) \text {. }
$$

The improvement of these two corrections on our predicted $\delta V_{1 p-1 n}$ is about $5 \mathrm{keV}$. Although the two contributions are small, but with more understanding of the symmetry energy of the nucleus, we believe that these contributes will become more important in the future.

In order to describe the nuclear mass obtained by our theory vividly, we compare the average RMSD of the nuclear mass with the experimental data to represent the difference, and the formula is as follows:

$$
\sigma=\sqrt{\frac{1}{n} \sum_{i=1}^{n}\left(M_{i}^{e x p}-M_{i}^{c a l}\right)^{2}}
$$

The RMSD is about $150 \mathrm{Kev}$. In Figure 2 we show deviations (in units of $\mathrm{keV}$ ) between our calculated $\delta V_{1 p-1 n}^{c a l}$ by applying Equations (6) and those experimental data of binding energies compiled in AME2012 [20]. It can be seen that the RMSDs of these $\delta V_{1 p-1 n}$ decrease with $A$. The description is better in the medium mass nucleus and heavy nucleus.

As early as 1960s, the nuclear structure theory predicts the existence of a number of new elements in the long life near the proton number $Z=114$ and neutron number $N=184$ (i.e. island of super heavy nuclei) and the island of super heavy nuclear plays an important role in the entire nuclear physics field. So for the heavy nuclei, we obtain another formula to describe the mass and it fits more closely with the experimental data. And in order to achieve better result, the different parameters are given between even-even nuclei and odd-odd nuclei, the formula is as follows:

$$
V_{1 p-1 n}(A)=\frac{a}{A^{2}}+\frac{b}{A}+c
$$

\begin{tabular}{rccc}
\hline Parameter & $a$ & $b$ & $c$ \\
\hline Even-even & -9464 & 146.3 & -0.06435 \\
Odd-odd & 46000 & -324.1 & 0.9124 \\
\hline
\end{tabular}




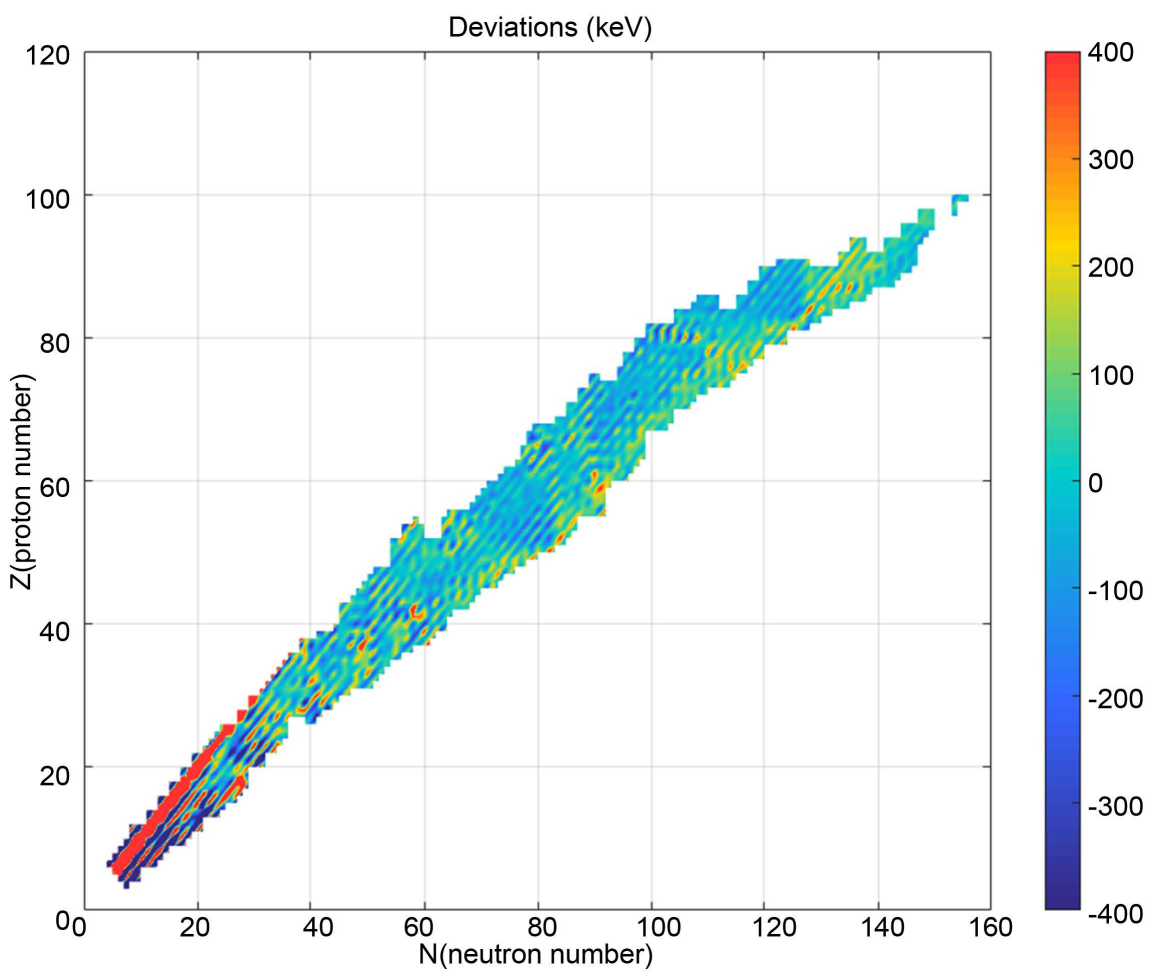

Figure 2. (Color online) Deviations (in units of $\mathrm{keV}$ ) of our calculated $\delta V_{1 p-1 n}^{\text {cal }}$ by using Equations (6) with respect to those extracted from experimental binding energies [Equation (4)], for the nuclei with $A \geq 16$.

When we use the Equation (6) to describe the nuclear masses, the RMSD is about $150 \mathrm{Kev}$, but if we try the Equation (7) where $A>200$, the RMSD is 119 $\mathrm{Kev}$, it shows that our formula of heavy nuclei is more accurate.

Figure 3 displays the difference between the experimental values and calculated values, we compare it with Ref [21], one can see that our result is better.

\section{Mass Predictions}

Through above study, we find our formula has a good performance in describing the nuclear masses. In this section, we use our formula and the residual proton-neutron interaction to predict the nuclear mass not obtained in the experiment. Based on the Equation (4), we can obtain

$$
M(Z, N)=M(Z-1, N)+M(Z, N-1)-M(Z-1, N-1)+\overline{\delta V_{1 p-1 n}}(A) .
$$

The unknown mass $M(Z, N)$ is predicted by using the three nuclei masses around it and the $\delta V_{1 p-1 n}(Z, N)$ we empirical obtained.

Now let's focus on a few examples of our predictions. Table 1 shows mass excess of some nuclei are not predictive in ame 2003 or ame 2012 databases. These unknown masses are important not only in the context of astrophysics, but also in the nuclear structure. Interestingly, our predicted values show good in comparison with the experimental results. For ${ }^{182} \mathrm{Lu}$, the deviation of our predicted masses from the value in AME2012 is only $\sim 63 \mathrm{keV}$. Three additional 


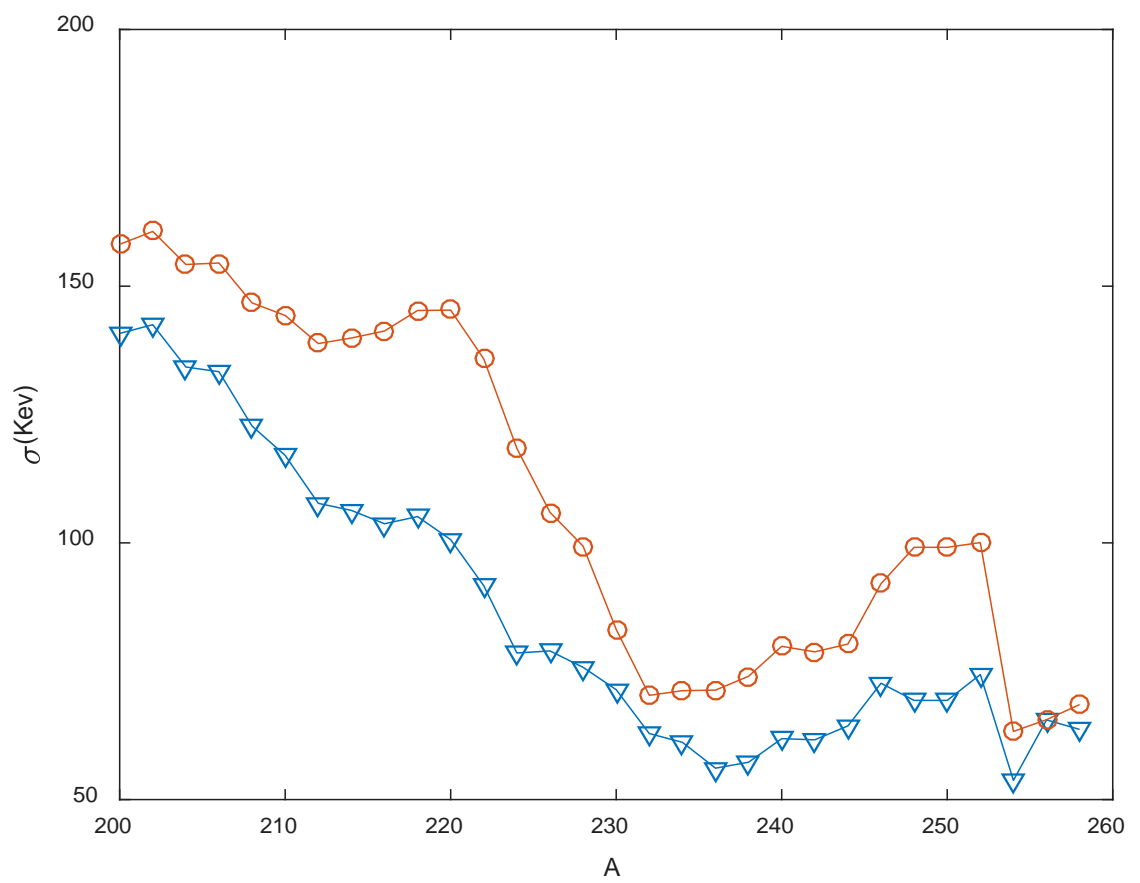

(a)

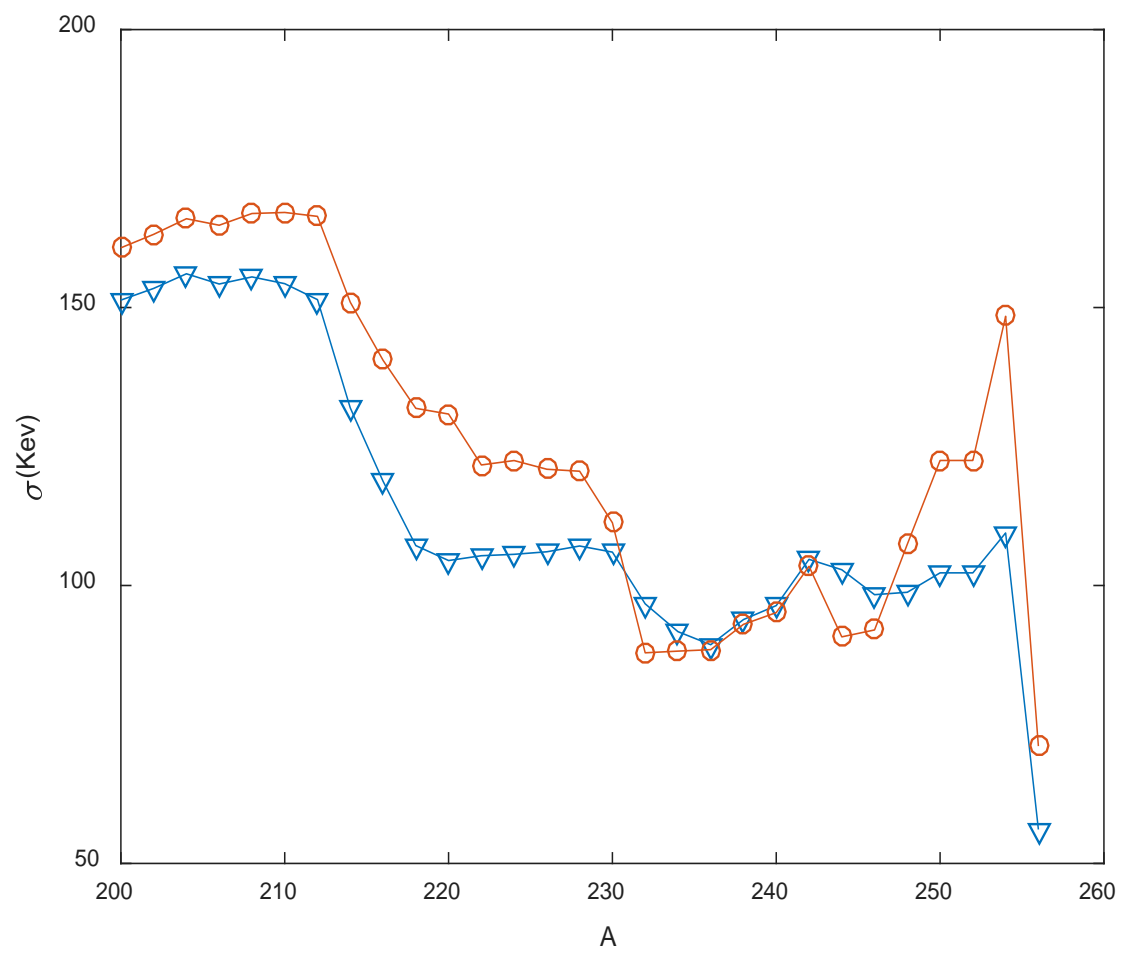

(b)

Figure 3. Shows the RMSDs of even-A nuclei. (a) represents the odd-odd nuclei; (b) represents the even-even nuclei. We obtain the even-A nuclear masses from some experimentally known nuclear masses and the residual proton-neutron interactions formula. Comparing calculated values with the AME2012 databases obtain the RMSDs. The triangles are plotted by using the RMSDs of our calculated values. The circles are plotted by using the formula in Ref [21]. 
Table 1. Mass excess of some mass nuclei with us and predicted results in the AME2003 database and the AME2012 databsae. (keV).

\begin{tabular}{cccc}
\hline Nucleus & AME2003 & AME2012 & $M^{\text {pred }}$ \\
\hline${ }^{52} \mathrm{Ni}$ & $-22,650$ & $-23,470$ & $-23,187$ \\
${ }^{74} \mathrm{Sr}$ & $-40,700$ & $-40,830$ & $-40,952$ \\
${ }^{86} \mathrm{As}$ & $-59,150$ & $-58,962$ & $-58,316$ \\
${ }^{98} \mathrm{Kr}$ & $-44,800$ & $-44,310$ & $-44,555$ \\
${ }^{126} \mathrm{Pr}$ & $-60,260$ & $-60,320$ & $-60,573$ \\
${ }^{148} \mathrm{Tm}$ & $-39,270$ & $-38,765$ & $-38,713$ \\
${ }^{164} \mathrm{Re}$ & $-27,640$ & $-27,523$ & $-27,422$ \\
${ }^{182} \mathrm{Lu}$ & $-41,880$ & $-41,880$ & $-41,817$ \\
${ }^{190} \mathrm{At}$ & null & null & 10,290 \\
${ }^{200} \mathrm{Ir}$ & null & $-21,611$ & $-21,693$ \\
${ }^{202} \mathrm{Pt}$ & $-22,600$ & $-22,692$ & $-22,592$ \\
${ }^{224} \mathrm{~Np}$ & null & 31,876 & 31,793 \\
${ }^{232} \mathrm{Am}$ & 43,400 & 43,268 & 43,376 \\
${ }^{272} \mathrm{Mt}$ & 133,890 & 133,582 & 133,671 \\
${ }^{286} \mathrm{Ed}$ & 168,120 & 169,725 & 169,700 \\
\hline
\end{tabular}

nuclei are ${ }^{202} \mathrm{Pt},{ }^{232} \mathrm{Am}$ and ${ }^{286} \mathrm{Ed}$, the differences between our predicted values and those in AME2012 are approximately $100 \mathrm{keV}$. It seems our formula shows a great accuracy and can be used predict nuclear masses.

\section{Discussion and Conclusions}

In this paper, we obtain the residual proton-neutron interactions formula to describe and predict the mass of even-A nuclei. In order to improve the accuracy of the $\delta V_{1 p-1 n}$, we use the average value of the $\delta V_{1 p-1 n}$ (denoted as $\overline{\delta V_{1 p-1 n}}$ modification) and introduce two modifications.

For further understanding of the super heavy nuclei, we use another formula to describe the $\delta V_{1 p-1 n}$, and its results fit the experiment data more accurate, one can see that the RMSD decreases considerably.

Then we investigate the predictive power of these new formulas by numerical experiments. They are competitive with other local mass relations. The deviation of predicted results from experimental values is less compared with other models.

Based on results so far, our method of studying the neighboring nuclei has a good performance. We can predict other unknown masses by using our empirical formula to provide useful reference points for experimental physics.

\section{Acknowledgements}

The author would like to thank G.Y.Gao for reading and commenting of this 
paper.

\section{References}

[1] Von Weizsäcker, C.F. (1935) Zur Theorie der Kernmassen. Zeitschrift für Physik, 96, 431. https://doi.org/10.1007/BF01337700

[2] Duflo J. and Zuker, A.P. (1995) Microscopic Mass Formulas. Physical Review C, 52, R23. https://doi.org/10.1103/PhysRevC.52.R23

[3] Möller P., Myers W. D., Sagawa, H. and Yoshida, S. (2012) New Finite-Range Droplet Mass Model and Equation-of-State Parameters. Physical Review Letters, 108, 052501. https://doi.org/10.1103/PhysRevLett.108.052501

[4] Wang N., Liang Z. Y., Liu, M. and Wu, X. Z. (2010) Mirror Nuclei Constraint in Nuclear Mass Formula. Physical Review C, 82, 044304. https://doi.org/10.1103/PhysRevC.82.044304

[5] Wang N., Liu, M. and Wu, X. Z. (2010) Modification of Nuclear Mass Formula by Considering Isospin Effects. Physical Review C, 81, 044322.

https://doi.org/10.1103/PhysRevC.81.044322

[6] Mendoza-Temis, J., Hirsch, J. G. and Zuker, A. P. (2010) The Anatomy of the Simplest Duflo-Zuker Mass Formula. Nuclear Physics A, 843, 14. https://doi.org/10.1016/j.nuclphysa.2010.05.055

[7] De Shalit A. and Goldhaber, M. (1953) Mixed Configurations in Nuclei. Physical Review Journals Archive, 92, 1211. https://doi.org/10.1103/PhysRev.92.1211

[8] Federman P. and Pittel, S. (1977) Towards a Unified Microscopic Description of Nuclear Deformation. Physics Letters B, 69, 385.

https://doi.org/10.1016/0370-2693(77)90825-5

[9] Casten R. F. and Zamfir, N. V. J. (1996) The Evolution of Nuclear Structure: The $N_{\mathrm{p}} N_{\mathrm{n}}$ Scheme and Related Correlations. Journal of Physics G: Nuclear and Particle Physics, 22, 1521. https://doi.org/10.1088/0954-3899/22/11/002

[10] Talmi, I. (1962) Effective Interactions and Coupling Schemes in Nuclei. Reviews of Modern Physics, 34, 704. https://doi.org/10.1103/RevModPhys.34.704

[11] Brenner, D.S., Wesselborg, C., Casten, R.F., Warner, D.D. and Zhang, J.Y. (1990) Empirical p-n Interactions: Global Trends, Configuration Sensitivity and $N=Z$ Enhancements. Physics Letters B, 243, 1 . https://doi.org/10.1016/0370-2693(90)90945-3

[12] Mouze, G., Bidegainberry, S., Rocaboy, A. and Ythier, C. (1993) The Neutron-Proton Interaction Energy of the Valence Nucleons. Il Nuovo Cimento A (1965-1970), 106, 885.

[13] Gao, Z.C., Chen, Y.S. and Meng, J. (2001) Garvey-Kelson Mass Relations and n-p Interaction. Chinese Physics Letters, 18, 1186.

https://doi.org/10.1088/0256-307X/18/9/310

[14] Cakirli R. B., Brenner D. S., Casten, R. F. and Millman, E. A. (2005) Proton-Neutron Interactions and the New Atomic Masses. Physical Review Letters, 94, 092501. https://doi.org/10.1103/PhysRevLett.94.092501

[15] Cakirli R. B. and Casten, R. F. (2006) Direct Empirical Correlation between Proton-Neutron Interaction Strengths and the Growth of Collectivity in Nuclei. Physical Review Letters, 96, 132501. https://doi.org/10.1103/PhysRevLett.96.132501

[16] Breitenfeldt M. et al., (2010) Approaching the $N=82$ Shell Closure with Mass Measurements of Ag and Cd Isotopes. Physical Review C, 81, 034313. https://doi.org/10.1103/PhysRevC.81.034313 
[17] Jiao, B.B. (2017) Description and Prediction of Even-A Nuclear Masses Based on Residual Proton-Neutron Interactions. https://arxiv.org/abs/1706.08686

[18] Garvey, G. T. and Kelson, I. (1966) New Nuclidic Mass Relationship. Physical Review Letters, 16, 197. https://doi.org/10.1103/PhysRevLett.16.197

[19] Garvey G. T., Gerace W. J., Jaffe R. L., Talmi, I. and Kelson, I. (1969) Set of Nuclear-Mass Relations and a Resultant Mass Table. Reviews of Modern Physics, 41, S1. https://doi.org/10.1103/RevModPhys.41.S1

[20] Wang, M., Audi, G., Wapstra, A.H., et al. (2012) The Ame2012 Atomic Mass Evaluation. Chinese Physics C, 36, 1603.

[21] Fu, G.J., Lei, Y., Jiang, H., et al. (2011) Description and Evaluation of Nuclear Masses Based on Residual Proton-Neutron Interactions. Physical Review C, 84, 034311. https://doi.org/10.1103/PhysRevC.84.034311 